\title{
An optimal control approach to public investments for unemployment reduction
}

\author{
Paolo Di Giamberardino \\ Dept. of Computer, Control and Management Engineering \\ Sapienza University of Rome, Rome, Italy \\ paolo.digiamberardino@uniroma1.it \\ Tommaso Belvedere \\ Dept. of Computer, Control and Management Engineering \\ Sapienza University of Rome, Rome, Italy \\ belvedere.1705326@studenti.uniroma1.it
}

\author{
Barbara Bazzana \\ Dept. of Computer, Control and Management Engineering \\ Sapienza University of Rome, Rome, Italy \\ bazzana.barbara@gmail.com \\ Daniela Iacoviello \\ Dept. of Computer, Control and Management Engineering \\ Sapienza University of Rome, Rome, Italy \\ daniela.iacoviello@uniroma1.it
}

\begin{abstract}
The paper deals with the modelling and the control of a job market dynamics which considers unemployed individuals and two classes of jobs: a temporary one, characterised by a lower quality of economical treatment and/or long duration assurance for the workers, and a regular one, more stable and economically more satisfactory. For each of the two classes, the active workers as well as the vacancies are considered. Control actions are introduced, representing different government efforts devoted to the quantity and the quality improvements of the work. Choices in the model are discussed and compared with literature. The numerical results of some simulations are reported to better put in evidence the results obtained.
\end{abstract}

Keywords-Job market; unemployment; optimal control

\section{INTRODUCTION}

Despite the different relevance that it has on the various nations, unemployment is a problem that affects all the Countries. Political solutions are adopted by all Governments to reduce the negative impact that the lack of job positions has on the society. Clearly, such solutions have a high cost in terms of public investments, with consequent relevant impact on the local economies.

The availability of a model for the dynamics of the job market can help both for prevision, as in [1], and for the design of an effective action, [2], [3], [4].

The majority of the models proposed in literature introduces a relationship between the unemployed people and the vacancies which strongly recalls the one between susceptible and infected individuals in the epidemic spreads: people are hired with the same logic as an individual becomes infected. In fact, an unemployed person may become an active worker when he meets a vacancy in the same way as an individual gets an infection after the contact with an infected subject. This means that it is possible to transfer the large knowledge on the epidemic diseases to the unemployment disease.

Since in epidemic context it is common to make reference to optimal control techniques to design suitable and effective actions keeping the control effort as low as possible, as in [5], [6], [7], [8], [9], [10], it comes straightforward to extend this technique for the problem of containment of unemployment.
For the specific problem at hands, in [4], [11], optimal controls are designed and their effectiveness is discussed.

In the present work, the model adopted in [11] is considered, with some modifications introduced to include additional characteristics. After the description and the discussion of such a mathematical model in Section II, the optimal control problem is formulated in Section III, trying to maximise the number of employed individuals in the best job positions. Numerical results are finally reported in Section IV to illustrate the effectiveness of the approach.

\section{The Mathematical Model}

The mathematical model describing the unemployedemployed-vacancies dynamics is here defined.

The starting point is the model introduced in [11], which is here slightly modified to take into account some additional possible transitions and influences between classes.

As in [11], the unemployed persons are considered in the class $U$. The working people are divided into two classes, according to the stability and the duration of the employment: a regular full time occupation characterises the class of employed persons in $R$, while temporary or part-time jobs define the class $T$. In the same way, the vacancies are also divided into two classes according to the kind of job for which a person is requested: temporary or part time available jobs are in the class of vacancies $V_{1}$, while the full time stable available jobs are in the $V_{2}$ class.

Then, the state variables adopted are: $U(t)$, the number of unemployed people at time $t ; R(t)$, the number of employed in the higher quality good jobs at time $t ; T(t)$, the number of employed in the lower quality jobs at time $t ; V_{1}(t)$ and $V_{2}(t)$ the number of vacancies in the low and high quality jobs respectively.

On the basis of the previous considerations, the dynamical 
model adopted is

$$
\begin{aligned}
\dot{U}= & \Lambda-\left(1+u_{1}\right) k_{1} U V_{1}-\left(1+u_{2}\right) k_{2} U V_{2} \\
& -\alpha_{1} U+\gamma_{1} T+\gamma_{2} R \\
\dot{T}= & \left(1+u_{1}\right) k_{1} U V_{1}-k_{3} T V_{2}-\left(\alpha_{2}+\gamma_{1}\right) T \\
\dot{R}= & \left(1+u_{2}\right) k_{2} U V_{2}-\left(\alpha_{3}+\gamma_{2}\right) R+k_{3} T V_{2} \\
\dot{V_{1}=} & -\left(1+u_{1}\right) k_{1} U V_{1}+k_{3} T V_{2}+\varepsilon_{1} \alpha_{2} T+\eta_{1} \gamma_{1} T \\
& +\left(1+u_{3}\right) \phi_{1} U-\delta_{1} V_{1} \\
\dot{V}_{2}= & -\left(1+u_{2}\right) k_{2} U V_{2}-k_{3} T V_{2}+\varepsilon_{2} \alpha_{3} R+\eta_{2} \gamma_{2} R \\
& +\left(1+u_{4}\right) \phi_{2} U-\delta_{2} V_{2}
\end{aligned}
$$

which can be represented, for sake of simplicity, also in the compact form

$$
\dot{x}=F(x, u)
$$

once the state vector $x=\left(\begin{array}{lllll}U & T & R & V_{1} & V_{2}\end{array}\right)^{T}$ and the control vector $u=\left(\begin{array}{llll}u_{1} & u_{2} & u_{3} & u_{4}\end{array}\right)^{T}$ are introduced.

The external inputs considered are added to natural relationships of the uncontrolled model, representing the effects of economic actions aiming at reducing the unemployed individuals or, equivalently, to increment the number of active workers:

$u_{1}$, which helps in providing temporary employment;

$u_{2}$, which helps in providing regular employment;

$u_{3}$ which provides new temporary vacancies;

$u_{4}$ which provides new regular vacancies.

In (1) the parameters have the following meanings:

$\Lambda$ : rate at which the number of unemployed persons increases for independent external actions;

$k_{1}$ : rate of temporary employment of unemployed persons;

$k_{2}$ : rate of regular employment of unemployed persons;

$k_{3}$ : rate of regular employment of temporary one;

$\alpha_{1}$ : rate of migration/death of unemployed people;

$\alpha_{2}$ : rate of retirement/death of temporarily employed persons;

$\alpha_{3}$ : rate of retirement/death of regularly employed persons;

$\gamma_{1}$ : rate at which people with temporary jobs are fired;

$\gamma_{2}$ : rate at which people with regular jobs are fired;

$\delta_{1}$ : rate of decrement of temporary vacancies (lack of funds);

$\delta_{2}$ : rate of decrement of regular vacancies (lack of funds);

$\phi_{1}$ : rate of creation of new temporary vacancies;

$\phi_{2}$ : rate of creation of new regular vacancies;

$\varepsilon_{1}$ : fraction of temporary job positions which become available after workers retirement;

$\varepsilon_{2}$ : fraction of regular job positions which become available after workers retirement;

$\eta_{1}$ : fraction of temporary job positions which become available after worker dismissal;

$\eta_{2}$ : fraction of regular job positions which become available after worker dismissal.

Fractions $0 \leq \varepsilon_{1} \leq 1$ and $0 \leq \varepsilon_{2} \leq 1$ are here introduced to model the fact that not all the retirements may correspond to a new recruitment: it can be dependent on the economic condition of the population considered; so, it is here supposed that only the fraction $\varepsilon_{1}$ of retirements among temporary jobs corresponds to new temporary vacancies, and the same for $\varepsilon_{2}$ for the regular jobs. A similar consideration can be performed for fired individuals. In the model (1) it is considered that only a fraction $0 \leq \eta_{1} \leq 1$ of temporary job positions occupied by fired persons returns to be available as temporary vacancy as well as only a fraction $0 \leq \eta_{2} \leq 1$ of regular jobs returns as a vacancy after firing regular workers.

Dynamics (1) contains different kinds of terms with hybrid relationships between them. In fact, while $U, T$ and $R$ represents individuals, $V_{1}$ and $V_{2}$ are job vacancies; moreover, a term like $k_{1} U V_{1}$ represents the interaction between individuals $U$ and jobs $V_{1}$, producing modifications both in individuals dynamics $\dot{T}$ and in job ones, $\dot{V}_{1}$. Setting $P=U+T+R$ and making use of (1), the dynamics

$$
\dot{P}=\Lambda-\alpha_{1} P-\left(\alpha_{2}-\alpha_{1}\right) T-\left(\alpha_{3}-\alpha_{1}\right) R
$$

represents the evolution of all the people. The first observation is that it does not depend directly on the control action: people increase according to the input rate $\Lambda$ and decrease due to death/retirement rates $\alpha_{1}, \alpha_{2}$ and $\alpha_{3}$ and the different classes of people. More precisely, (3) has an exponentially stable dynamics with external actions given by the input rate $\Lambda$ and the two effects of $T$ and $R$, which can be positive or negative depending on the difference between the values of the coefficients $\alpha_{i}$. Since these coefficients denote the rate of retirements for $T\left(\alpha_{2}\right)$ and for $R\left(\alpha_{3}\right)$ and the emigrations for class $U\left(\alpha_{1}\right)$, it can be assumed that $\alpha_{i}>\alpha_{1}, i=2,3$, since normally retirements are greater than emigrations.

The control acts on the number of employed persons $T$ and $R$. This is reasonable, since control actions are defined in order to improve the performances of the job market. Moreover, the variables $T$ and $R$, the employed persons, can also be associated to the number of occupied job positions. This means that, defining the new variable $W=T+R+V_{1}+V_{2}$, which denotes the number of all the job positions, its dynamics is

$$
\begin{aligned}
\dot{W}= & \left(\left(1+u_{3}\right) \phi_{1}+\left(1+u_{4}\right) \phi_{2}\right) U-\delta_{1} V_{1}-\delta_{2} V_{2} \\
& -\left(\left(1-\varepsilon_{1}\right) \alpha_{2}+\left(1-\eta_{1}\right) \gamma_{1}\right) T \\
& -\left(\left(1-\varepsilon_{2}\right) \alpha_{3}+\left(1-\eta_{2}\right) \gamma_{2}\right) R
\end{aligned}
$$

From (4) it is clear the reason of the introduction of the terms $\phi_{1} U$ and $\phi_{2} U$ in the $V_{1}$ and $V_{2}$ dynamics: without them, in the uncontrolled case the total number of job positions would decrease independently from the number of individuals in the society, unrealistic in a real context. In fact, it is quite easy to compute the equilibrium points for the system (1) when $\phi_{1}=\phi_{2}=0$. They are the solutions of the system

$$
\begin{aligned}
\Lambda-k_{1} U V_{1}-k_{2} U V_{2}-\alpha_{1} U+\gamma_{1} T+\gamma_{2} R & =0 \\
k_{1} U V_{1}-k_{3} T V_{2}-\left(\alpha_{2}+\gamma_{1}\right) T & =0 \\
k_{2} U V_{2}+k_{3} T V_{2}-\left(\alpha_{3}+\gamma_{2}\right) R & =0 \\
-k_{1} U V_{1}+k_{3} T V_{2}+\left(\varepsilon_{1} \alpha_{2}+\eta_{1} \gamma_{1}\right) T+\phi_{1} U-\delta_{1} V_{1} & =0 \\
-k_{2} U V_{2}-k_{3} T V_{2}+\left(\varepsilon_{2} \alpha_{3}+\eta_{2} \gamma_{2}\right) R+\phi_{2} U-\delta_{2} V_{2} & =0
\end{aligned}
$$

once $\phi_{1}=0$ and $\phi_{2}=0$ are set. Only one admissible point is obtained, $x_{e}=\left(\begin{array}{lllll}\frac{\Lambda}{\alpha_{1}} & 0 & 0 & 0 & 0\end{array}\right)^{T}$. Local stability can be 
studied by computing the Jacobian matrix of (1) evaluated at the equilibrium point. One gets

$$
J=\left(\begin{array}{ccccc}
-\alpha_{1} & \gamma_{1} & \gamma_{2} & -k_{1} \frac{\Lambda}{\alpha_{1}} & -k_{2} \frac{\Lambda}{\alpha_{1}} \\
0 & -\left(\alpha_{2}+\gamma_{1}\right) & 0 & k_{1} \frac{\Lambda}{\alpha_{1}} & 0 \\
0 & 0 & -\left(\alpha_{3}+\gamma_{2}\right) & 0 & k_{2} \frac{\Lambda}{\alpha_{1}} \\
0 & \left(\varepsilon \alpha_{2}+\eta \gamma_{1}\right) & 0 & -k_{1} \frac{\Lambda}{\alpha_{1}}-\delta_{1} & 0 \\
0 & 0 & \left(\varepsilon \alpha_{3}+\eta \gamma_{2}\right) & 0 & -k_{2} \frac{\Lambda}{\alpha_{1}}-\delta_{2}
\end{array}\right)
$$

The eigenvalues of (5) can be obtained after some easy manipulations. One is given by $\lambda_{1}=-\alpha_{1}$, while the other four are the solutions of the two second order equations

$$
\begin{aligned}
& \lambda^{2}+\left(\alpha_{2}+\gamma_{1}+\delta_{1}+k_{1} \frac{\Lambda}{\alpha_{1}}\right) \lambda+ \\
& \left(k_{1} \frac{\Lambda}{\alpha_{1}}\left((1-\varepsilon) \alpha_{2}+(1-\eta) \gamma_{1}\right)+\delta_{1}\left(\alpha_{2}+\gamma_{1}\right)\right)=0 \\
& \lambda^{2}+\left(\alpha_{3}+\gamma_{2}+\delta_{2}+k_{2} \frac{\Lambda}{\alpha_{1}}\right) \lambda+ \\
& \left(k_{2} \frac{\Lambda}{\alpha_{1}}\left((1-\varepsilon) \alpha_{3}+(1-\eta) \gamma_{2}\right)+\delta_{2}\left(\alpha_{3}+\gamma_{2}\right)\right)=0
\end{aligned}
$$

Both of them have all the coefficients positive, so that for the Descartes' rule of signs, they both have two solutions with negative real part. It is possible to conclude that the equilibrium point $x_{e}$ is locally asymptotically stable. This means that for this model, without any external action, the population at steady state is composed only by unemployed since there are neither vacancies nor occupied persons and this situation is definitely unrealistic.

The additional terms $\phi_{1} U$ and $\phi_{2} U$ define a relationship between the dimension of the population and the possible job positions so that in presence of unemployed not only it is impossible to have no vacancies, but the more are the unemployed the more are the vacancies. It is interesting to note that this choice has the consequence of keeping the two job classes separated, so that the variations of vacancies for each kind of job do not depend on the quantity of the other class. This can be seen by setting $W_{1}=T+V_{1}$, corresponding to all the available temporary jobs, occupied or not by a worker, and computing its time variation

$$
\dot{W}_{1}=\left(1+u_{3}\right) \phi_{1} U-\left(\left(1-\varepsilon_{1}\right) \alpha_{2}+\left(1-\eta_{1}\right) \gamma_{1}\right) T-\delta_{1} V_{1}
$$

from which it is possible to conclude that in the uncontrolled dynamics with $\phi_{1}=0$, variation in (6) is negative and depends only on the temporary workers $T$ and on the temporary vacancies $V_{1}$. Introducing $\phi_{1}$ and $\phi_{2}$, as well as considering the control actions, the $W_{1}$ dynamics is still independent from the other type of jobs, $R$ and $V_{1}$, and from any other control but $u_{3}$. Same considerations can be performed defining $W_{2}=R+V_{2}$ and studying the dynamics of the regular jobs, occupied or available. This fact reflects also on the equilibrium points. In fact, after some computation, it is possible to conclude that if $\phi_{1} \neq 0$ and $\phi_{2}=0$ are set, the system has one stable equilibrium point with the structure $x_{e}\left(\phi_{1}\right)=\left(\begin{array}{lllll}U_{e} & T_{e} & 0 & V_{1, e} & 0\end{array}\right)^{T}$ while, for $\phi_{1}=0$ and $\phi_{2} \neq 0$, one has $x_{e}\left(\phi_{2}\right)=\left(\begin{array}{lllll}U_{e} & 0 & R_{e} & 0 & V_{2, e}\end{array}\right)^{T}$.

\section{The Control Problem Formulation}

The goal of a control action is to have the maximum number of workers with respect to the total population. Clearly, better results require higher control efforts and then a form of limitation of the control has to be introduced corresponding to a limited quantity of resources.

A control design framework in which the maximum performances are required under some input constraints is the optimal control one. In fact, the optimal control for a given system is defined as the control which minimises (maximises) a given cost function under possible constraints on the final conditions, on the final time and on the control.

In [11] the cost function has been defined in order to minimise the number of unoccupied individuals minimising, at the same time, the control effort. It is

$$
J=\int_{0}^{t_{f}}\left(A_{1} U+\frac{1}{2}\left(A_{2} u_{1}^{2}+A_{3} u_{2}^{2}+A_{4} u_{3}^{2}+A_{5} u_{4}^{2}\right)\right) d t
$$

In the present work a different choice is performed. The idea at the basis of this approach is that, despite the mathematical model describes two kinds of jobs, one, the temporary one, clearly less attractive than the other one, the regular work, the control designed in [11] does not distinguish between the two types when aims at minimising the unemployed, no care which kind of job the worker will do. In order to take into account this difference, the same goal as the one in (7) is fixed but changing the idea of minimising the unemployed with the equivalent one of maximising the employed, being able, in this case, to assign different importance, and then different weights, to the two classes. The resulting cost function to be minimised is defined as

$$
J=\int_{0}^{t_{f}}\left(-A_{1} T-A_{2} R+\frac{1}{2} \sum_{i=1}^{4} B_{i} u_{i}^{2}\right) d t
$$

where the minus signs for the $T$ and $R$ terms are introduced to transform a maximization problem into a minimization one.

The controls are included in the cost function for limiting their effort; an explicit constraint is introduced for each control input, requiring that during the system evolution they must satisfy $0 \leq u_{i} \leq 1, i=1, \ldots, 4$. The initial condition for the state $x(0)$ is known and fixed, while the final state value is left free; the final time is supposed fixed. The solution can be computed defining firstly he Hamiltonian function $H=$ $L(x, u)+\lambda^{T} F(x, u)$ where $\lambda \in R^{5}$ are the costate variables, to be computed during the control problem solution. The optimal solution must satisfy the following necessary conditions:

$$
\dot{\lambda}=-\left.\frac{\partial H}{\partial x}\right|^{T} \quad \text { with } \quad \lambda\left(t_{f}\right)=0,\left.\quad \frac{\partial H}{\partial u}\right|^{T}=0
$$

From the control equation in (9) it is possible to compute the input as a function of the state and the costate.

In fact

$$
\frac{\partial H}{\partial u_{1}}=A_{2} u_{1}-k_{1}\left(\lambda_{1}+\lambda_{2}-\lambda_{4}\right) U V_{1}=0
$$


gives

$$
u_{1}=\frac{k_{1}}{A_{2}}\left(\lambda_{1}+\lambda_{2}-\lambda_{4}\right) U V_{1}=\Omega_{1}(x, \lambda)
$$

In the same way, from $\frac{\partial H}{\partial u_{i}}=0, i=2,3,4$, one has $u_{i}=$ $\Omega_{i}(x, \lambda), i=2,3,4$. Due to the presence of the bounds on the controls, the optimal solutions can be expressed as

$$
u_{i}=\min \left\{1, \max \left\{0, \Omega_{i}(x, \lambda)\right\}\right\}, \quad i=1, \ldots, 4
$$

Then, once the differential equations of the state and the costate, with their boundary conditions, are solved, the explicit expression of the control is determined. In the following Section IV some numerical results are reported and commented, to validate the proposed procedure.

\section{NumERICAL RESUlTS}

In next Subsection IV-A, some numerical results are firstly reported to better clarify the role of some of the parameters introduced in the model and already discussed in Section II. Then, in Subsecton IV-B, the optimal control is computed and simulated. The numerical values used for the simulations are reported in Table I. All the parameters that the model (1) has

TABLE I

VALUE OF THE PARAMETERS

\begin{tabular}{|c|c|c|c|c|}
\hline Parameter & Value & & Parameter & Value \\
\hline$\Lambda$ & 500 & & $k_{1}$ & $3 \cdot 10^{-6}$ \\
\hline$k_{2}$ & $2 \cdot 10^{-6}$ & & $k_{3}$ & $4.5 \cdot 10^{-6}$ \\
\hline$\gamma_{1}$ & $10^{-3}$ & & $\gamma_{2}$ & $2 \cdot 10^{-3}$ \\
\hline$\alpha_{1}$ & 0.004 & & $\alpha_{2}$ & 0.005 \\
\hline$\alpha_{3}$ & 0.006 & & $\phi_{1}$ & 0.22 \\
\hline$\phi_{2}$ & 0.20 & & $\delta_{1}$ & 0.09 \\
\hline$\delta_{2}$ & 0.05 & & $\varepsilon_{1}$ & 0.8 \\
\hline$\varepsilon_{2}$ & 0.8 & & $\eta_{1}$ & 0.2 \\
\hline$\eta_{2}$ & 0.2 & & & \\
\hline
\end{tabular}

in common with the one in [11] are chosen equal. The values of the additional parameters, the percentages $\varepsilon_{1}, \varepsilon_{2}, \eta_{1}$ and $\eta_{2}$, are set without introducing differences between the two types of works, temporary and regular, and on the basis of the idea that the probability that a job position became a vacancy after retirement is higher than the case of worker fired.

The initial conditions $x(0)$ for (1) are set following [11]: $x(0)=\left(\begin{array}{lllll}10^{5} & 2 \cdot 10^{3} & 10^{3} & 2 \cdot 10^{2} & 2 \cdot 10^{2}\end{array}\right)^{T}$.

\section{A. Model Parameters Effects}

A first set of numerical simulations is performed to validate the discussion reported in Section II about the presence of a natural vacancy creation depending on the number of unemployed individuals, given by the terms $\phi_{1} U$ and $\phi_{2} U$ in the uncontrolled dynamics, and the introduction of the percentages of vacancies created by retirements and dismissals, i.e. the coefficients $\varepsilon_{1}, \varepsilon_{2}, \eta_{1}, \eta_{2}$.

Clearly, the uncontrolled dynamics is here considered. So, first of all $\varepsilon_{1}=\varepsilon_{2}$ and $\eta_{1}=\eta_{2}$ are posed, to reduce the number of different simulations without losing a high level of generality. Then, four cases are considered for the parameters $\varepsilon_{i}$ and $\eta_{i}$, obtained combining the choices of a low (0.2) or a high (0.8) value for each of them. In order to better put in evidence the roles of such parameters in the vacancies dynamics with respect to the other effective contribution to the growth of the number of vacancies given by the terms $\phi_{1} U$ and $\phi_{2} U$, two cases are considered: one with $\phi_{1}=\phi_{2}=0$, to show the contribution of the parameters $\varepsilon_{i}$ and $\eta_{i}$ only, the other one with the values of $\phi_{1}$ and $\phi_{2}$ given in Table I. Figures 1-3 refer to the first of the two cases, while Figures 4-6 are related to the second one. The main observation is that, when the only contribution for restoring the number of the vacancies comes from the job positions left free after retirements or firing workers, the difference of the behaviours with respect of the percentages $\varepsilon_{1}, \varepsilon_{2}, \eta_{1}, \eta_{2}$ is very sensible; the total number of working people increases as the percentages does, Figure 1 , since the total job positions increase, Figure 2, due to the increment of the vacancies, Figure 3. On the other hand, in presence of a creation of new vacancies produced by $\phi_{1} U$ and $\phi_{2} U$, the higher contribution of these latter terms with respect to the restoration produced by the terms with $\varepsilon_{1}=\varepsilon_{2}$ and $\eta_{1}=\eta_{2}$ yields to a very low effects of such parameters on vacancy and job behaviours. In fact, in Figure 4 as well as in Figure 5 and Figure 6, the effect of the different values of the percentages $\varepsilon_{1}=\varepsilon_{2}$ and $\eta_{1}=\eta_{2}$ can be appreciated only after the fast transient due to the high initial number of unemployed individuals. 5

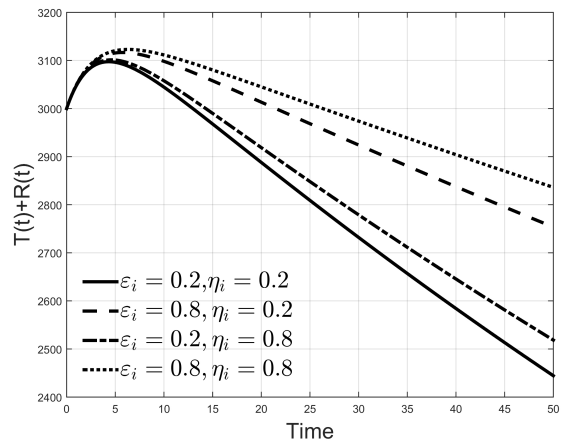

Fig. 1. Number of total workers for different values of $\varepsilon_{1}, \varepsilon_{2}, \eta_{1}, \eta_{2}$ and with $\phi_{1}=\phi_{2}=0$.

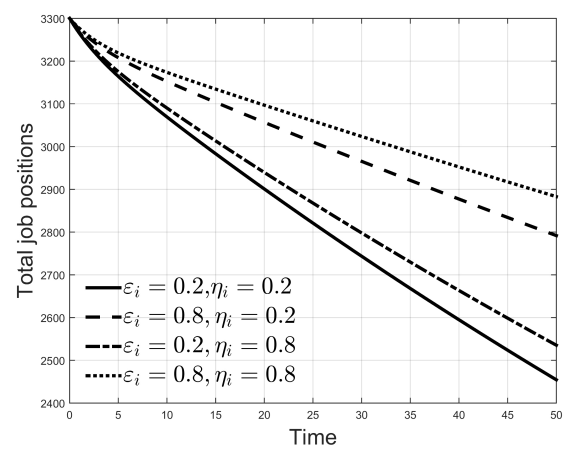

Fig. 2. Number of total job positions for different values of $\varepsilon_{1}, \varepsilon_{2}, \eta_{1}, \eta_{2}$ and with $\phi_{1}=\phi_{2}=0$. 


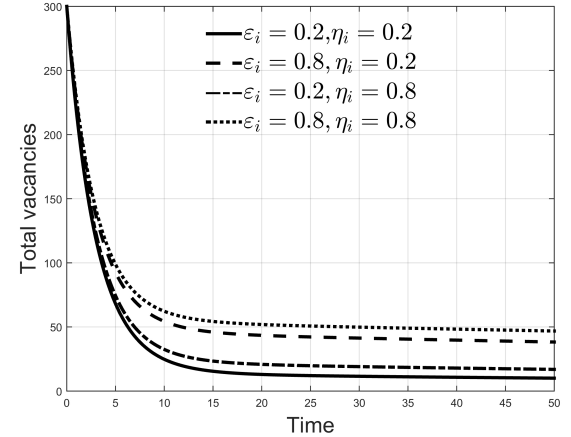

Fig. 3. Number of total vacancies for different values of $\varepsilon_{1}, \varepsilon_{2}, \eta_{1}, \eta_{2}$ and with $\phi_{1}=\phi_{2}=0$.

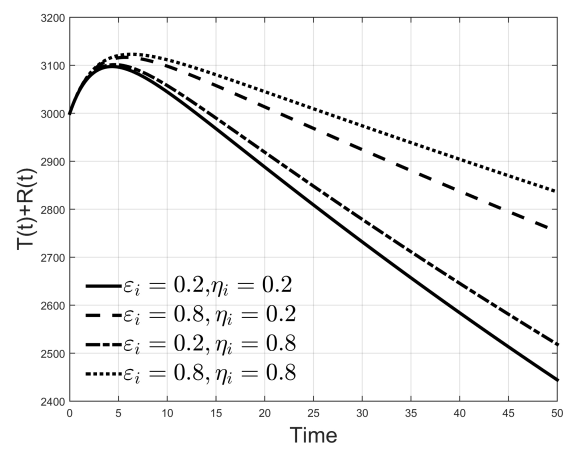

Fig. 4. Number of total workers for different values of $\varepsilon_{1}, \varepsilon_{2}, \eta_{1}, \eta_{2}$ and with $\phi_{1}=\phi_{2}$ as in Table I.

\section{B. Optimal Control Computation and Simulation}

For the computation of the optimal control problem defined in Section III, the values chosen for the weights $B_{i}$ present in (8) are [11] $B_{1}=10^{4}, B_{2}=10^{4}, B_{3}=3 \cdot 10^{4}$ and $B_{4}=4 \cdot 10^{4}$. For $A_{1}$ and $A_{2}$, three cases are considered.

i. $A_{1}=A_{2}=30$; in this case no preferences are indicated and the two types of works are considered equally;

ii. $\quad A_{1}=30$ and $A_{2}=300$; in this case a preference is given to the regular jobs, aiming at obtaining more regular jobs

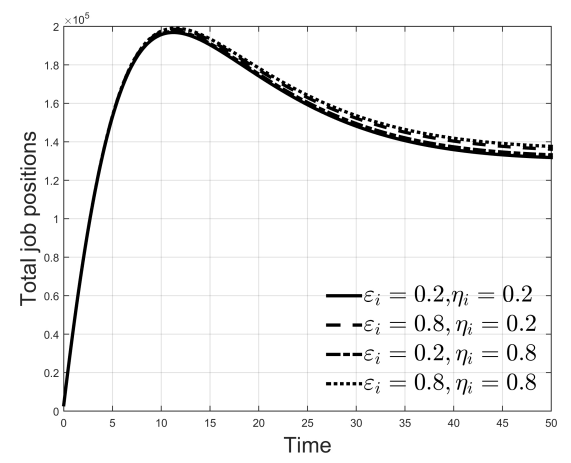

Fig. 5. Number of total job positions for different values of $\varepsilon_{1}, \varepsilon_{2}, \eta_{1}, \eta_{2}$ and with $\phi_{1}=\phi_{2}$ as in Table I.

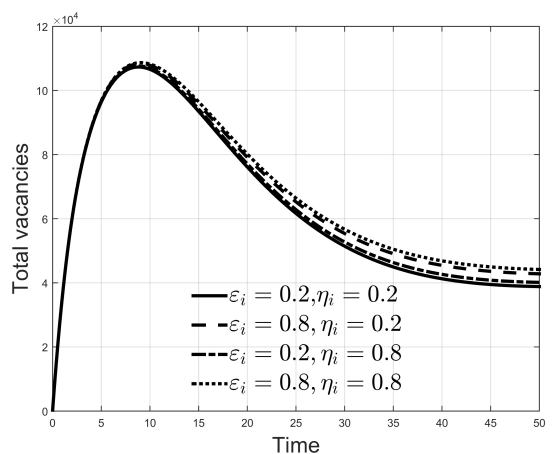

Fig. 6. Number of total vacancies for different values of $\varepsilon_{1}, \varepsilon_{2}, \eta_{1}, \eta_{2}$ and with $\phi_{1}=\phi_{2}$ as in Table I.

and less temporary ones with respect to the previous case; this is supposed to be a better situation for the workers;

iii. $A_{1}=30$ and $A_{2}=3000$, stressing the situation considered in $i$.

The results of the implementation of the three control laws computed according to the choices $i$, ii. and $i i i$. are reported in Figures 7-13. A first obvious observation is that the controlled cases are always better than the uncontrolled one. Moreover, as expected, increasing the value of the weight $B_{1}$, the number of regular jobs increases, Figure 8 , while, for the minimization/maximization requirements, the temporary ones have to decrease, as it can be seen in Figure 7. However, an unexpected effect is that once the time evolution of the total number of workers is considered, Figure 9, increasing the weight associated to the regular workers, a reduction is noticed, and the best performance is the one associated to an equally weighted jobs. In other words, from this model it seems that if a control action is applied to increment the number of workers, the requirement to force the better quality of the jobs produces a reduction of the total number of workers; it is interesting to notice that this effect does not correspond to a reduction of the control effort, as it can be seen in Figures 10-13, where the four controls are depicted.

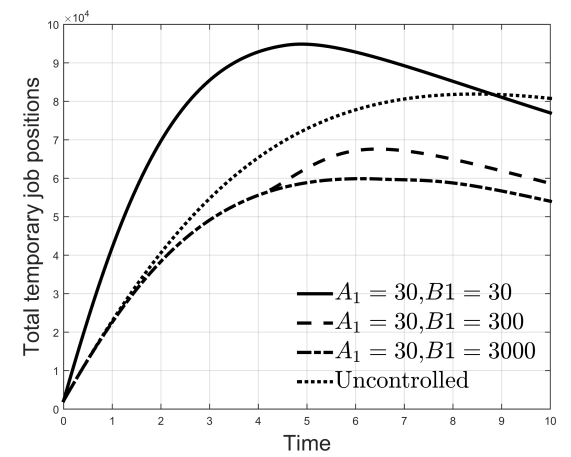

Fig. 7. Time evolution of all the temporary jobs (workers and vacancies). 


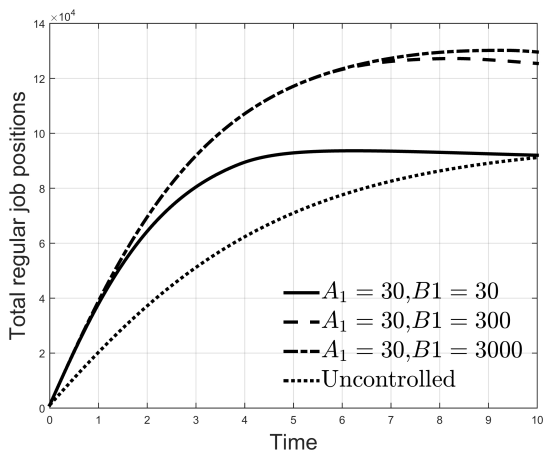

Fig. 8. Time evolution of all the regular jobs (workers and vacancies).

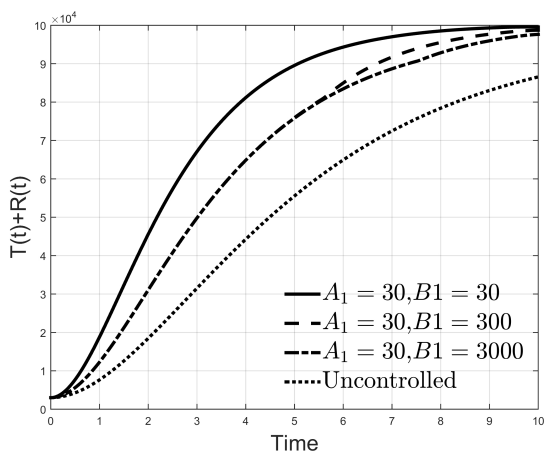

Fig. 9. Time evolution of all the active workers.

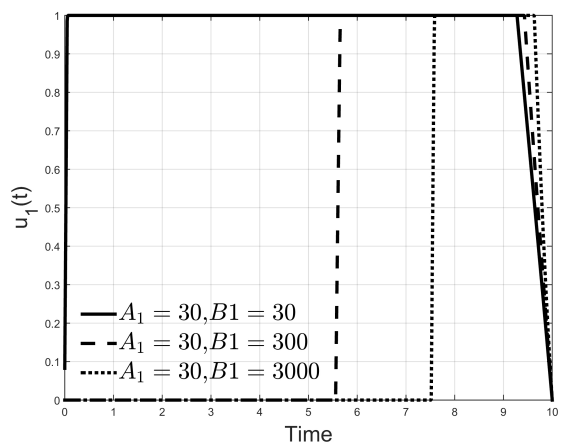

Fig. 10. Time evolution of the optimal control $u_{1}(t)$.

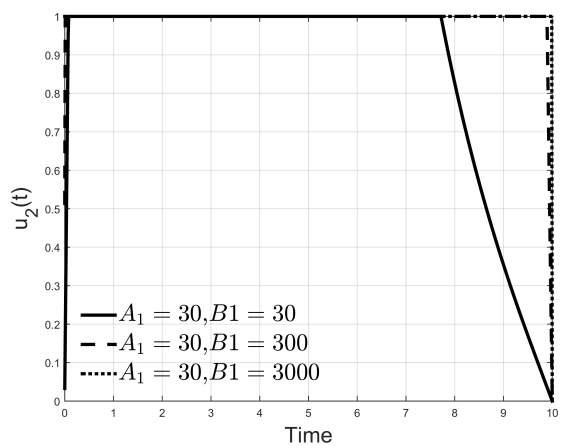

Fig. 11. Time evolution of the optimal control $u_{2}(t)$.

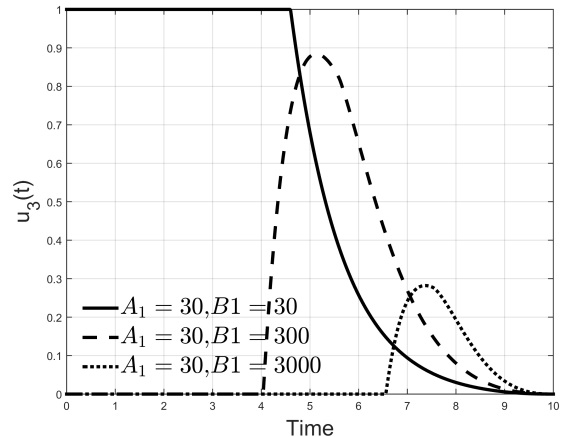

Fig. 12. Time evolution of the optimal control $u 3(t)$.

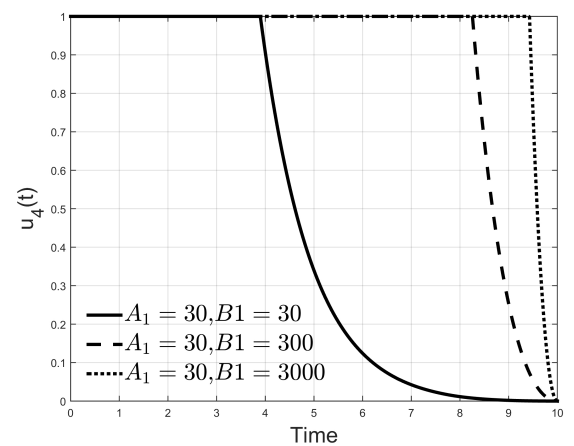

Fig. 13. Time evolution of the optimal control $u_{4}(t)$.

\section{REFERENCES}

[1] G. Wang and X. Zheng, "The unemployment rate forecast model basing on bp neural network," International Conference on Electronic Computer Technology, 2009.

[2] A. Misra and A. Singh, "A mathematical model for unemployment," Nonlinear Analysis: Real World Applications, vol. 12, 2011.

[3] A. Misra and A. Singh, "A delay mathematical model for the control of unemployment," Differential Equations and Dynamical Systems, vol. 21, no. 3, pp. 291?-307, 2011.

[4] S. B. Munoli and S. Gani, "Optimal control analysis of mathematical model for unemployment," Optimal Control Applications and Methods, vol. 37, no. 3, pp. 7984-806, 2016.

[5] H. Behncke, "Optimal control of deterministic epidemics," Optimal control applications and methods, vol. 21, 2000.

[6] P. Di Giamberardino, L. Compagnucci, C. D. Giorgi, and D. Iacoviello, "Modeling the effects of prevention and early diagnosis on HIV/AIDS infection diffusion," IEEE Trans. on Systems, Man and Cybernetics: Systems, 2018.

[7] P. Di Giamberardino and D. Iacoviello, "LQ control design for the containment of the HIV/AIDS diffusion," Control Engineering Practice vol. 77, 2018.

[8] P. Di Giamberardino and D. Iacoviello, "Optimal control to reduce the HIV/AIDS spread," Proceedings of the 22nd International Conference on System Theory, Control and Computing, 2018.

[9] P. Di Giamberardino and D. Iacoviello, "Modeling and control of an epidemic disease under possible complication," Proceedings of the 22 nd International Conference on System Theory, Control and Computing, pp. 67-72, 2018

[10] P. Di Giamberardino and D. Iacoviello, "Analysis, simulations and control of a new measles epidemic model," International Conference on Informatics in Control, Automation and Robotics, p. In press, 2019.

[11] S. B. Munoli, S. R. Gani, and S. R. Gani, "A mathematical approach to employment policies: An optimal control analysis," International Journal of Statistics and Systems, vol. 12, no. 3, pp. 594-565, 2017 